\title{
A BAYESIAN APPROACH TO MORPHOLOGICAL MODELS CHARACTERIZATION
}

\author{
Bruno Figliuzzi ${ }^{\bowtie, 1}$, Antoine MontauX-Lambert ${ }^{2}$, FranÇOis Willot ${ }^{1}$, Grégoire \\ NAUdin $^{2}$, Pierre Dupuis ${ }^{2}$, BERnARd QuerleuX $^{2}$ AND ETIENNe HugueT ${ }^{2}$ \\ ${ }^{1}$ Centre for Mathematical Morphology, Mines ParisTech - PSL Research University, Fontainebleau, 77300, \\ France, ${ }^{2}$ L'Oréal R\&I, Aulnay-sous-Bois, 93600, France \\ e-mail: bruno.figliuzzi@mines-paristech.fr, antoine.montaux@rd.loreal.com, \\ francois.willot@mines-paristech.fr, gregoire.naudin@ rd.loreal.com, pierre.dupuis@ rd.loreal.com, \\ bernard.querleux@rd.loreal.com, etienne.huguet@rd.loreal.com \\ (Received November 10, 2021; accepted November 26, 2021)
}

\begin{abstract}
Morphological models are commonly used to describe microstructures observed in heterogeneous materials. Usually, these models depend upon a set of parameters $\Theta$, that must be chosen carefully to match experimental observations conducted on the microstructure. A common approach to perform the parameters determination is to try to minimize an objective function, usually taken to be the discrepancy between measurements computed on the simulations and on the experimental observations, respectively. In this article, we present a Bayesian approach for determining the parameters of morphological models, based upon the definition of a posterior distribution for the parameters. A Monte Carlo Markov Chains (MCMC) algorithm is then used to generate samples from the posterior distribution and to identify a set of optimal parameters. We show on several examples that the Bayesian approach allows us to properly identify the optimal parameters of distinct morphological models and to identify potential correlations between the parameters of the models.
\end{abstract}

Keywords: Bayesian models, Monte Carlo Markov Chains algorithms, Morphological models.

\section{INTRODUCTION}

Materials used in industry often present a complex internal microstructure, which largely determines most of their physical properties at the macroscopic level (Jeulin, 1991; Ohser, 2009; Torquato, 2013; Moussaoui, 2018; 2019; Figliuzzi, 2019). A common way to carry out a quantitative study of the microstructure influence on the macroscopic properties of materials is to generate random microstructures that reproduce their geometrical characteristics (Moreaud, 2012; Wang, 2015). These simulated microstructures can subsequently be used to analyze the physical or mechanical properties of heterogeneous materials through extensive numerical simulations (Gasnier, 2015; Figliuzzi, 2016; Koishi, 2017; Bortolussi, 2018; Belhadj, 2018). This approach is particularly efficient to determine microstructures yielding optimized functional properties for some considered application.

Stochastic geometry models are often used to describe microstructures observed in heterogeneous materials (Chiu, 2013). Hence, microstructure simulation approaches based upon classical morphological models have drawn significant attention in the literature (Jeulin, 1991; 2012; 2017). A general introduction to this topic can be found in (Jeulin, 2021). Usually, the morphological models depend upon a set of parameters $\Theta$, that must be chosen carefully to match the experimental observations conducted on the material. A common approach to perform the parameters determination is to try to minimize an objective function, usually taken to be the discrepancy between measurements computed on the simulations and on the experimental observations, respectively. To that end, several methods have been proposed in the literature.

A classical approach is to minimize the objective function through a gradient descent algorithm. Gradient descent algorithms are first-order iterative optimization algorithms that seek local minima of a differentiable function by iteratively progressing toward steps proportional to the negative of the gradient in the parameters space. Gradient descent algorithms are very efficient to perform the minimization of strongly convex functionals and are extensively used in a number of fields. Nevertheless, several difficulties are associated to these methods when applied to the problem considered here. Firstly, for morphological models, the computation of the objective function gradient is often untrackable analytically, and one has therefore to rely on a finite difference scheme to evaluate the gradient at each iteration of the descent algorithm. At each step, this requires to compute at least $D+1$ evaluations of 
the objective function, $D$ being the dimension of the parameters space. In addition, the intrinsic statistical variability of the morphological models often leads to noisy estimates of the gradient. Secondly, the objective function that one seeks to minimize is often nonconvex, which means that a gradient descent algorithm will only converge toward a local minimum of the objective function.

Several alternatives to the gradient descent algorithm have been proposed in the literature. In (Wang, 2015) and (Figliuzzi, 2016) the authors notably propose to conduct the optimization procedure by employing the Nelder-Mead algorithm, a heuristic that can be used to determine the minimum or maximum of an objective function in a multidimensional space. It is a direct search method that only relies upon iterative evaluations of the objective function, and which can therefore be applied to problems for which the computation of the gradient is not trackable analytically.

In this article, we propose an approach based upon a Bayesian formulation of the problem, which allows us to define a posterior distribution for the parameters of the microstructure model depending on the experimental observations. A Monte Carlo Markov Chains (MCMC) algorithm allows us to generate samples from the posterior distribution. This approach presents several advantages when compared to other methods. In particular, the MCMC algorithm outputs samples that reproduce the actual posterior distribution, which enables detecting correlations between parameters of the model and quantifying the respective influences of the parameters.

\section{MATHEMATICAL MODEL}

\section{PROBLEM STATEMENT}

From now on, we assume that we dispose of a set of experimental images, or alternatively of a large experimental image of some materials microstructure. The experimental images can be transformed into binary images where the microstructure elements are represented by the value 1 (white) and the background by the value 0 (black). We will refer to these binary images as the observations $\mathscr{O}$. It is possible to extract measurements from the observations $\mathscr{O}$ by considering several statistical features, including the covariance, the granulometry or the granulometry of the complementary image:

- The covariance of a random set $A \subset \mathbb{R}^{D}$ is the function $C_{A}$ defined on $\mathbb{R}^{D} \times \mathbb{R}^{D}$ by

$$
C_{A}(x, x+h)=P\{x \in A, x+h \in A\},
$$

where $h$ is some vector of $\mathbb{R}^{D}$. The covariance of the set $A$ at a given point $x$ and for a distance $h$ is simply the probability that $x$ and $x+h$ both belong to $A$. For a stationary random set, the covariance is a function of the distance $h$ only:

$$
C_{A}(x, x+h)=C_{A}(h)
$$

If the set $A$ is ergodic, the covariance $C(h)$ can be estimated from the volume fraction of $A \cap A_{-h}$ to be

$$
C_{A}(h)=P\left\{x \in A \cap A_{-h}\right\}=V\left(A \cap A_{-h}\right),
$$

where $A_{-h}$ is the translation of the set $A$ by the vector $-h$. The covariance $C_{A}$ provides useful information about the spatial arrangement of the random set $A$. In particular, it can account for the presence of several scales in the studied set or for periodicity.

- The granulometry by openings of a random set $A$ provides a characterization of the size distribution of the elements of $A$. Let $K$ be a convex set. We consider the family of structuring elements $\left\{K_{\lambda}, \lambda>0\right\}$, where $K_{\lambda}=\lambda K$. The operator defined for all closed set $A \subset \mathbb{R}^{D}$ by

$$
\Phi_{\lambda}(A)=\left(A \ominus \check{K_{\lambda}}\right) \oplus K_{\lambda}
$$

where $\ominus$ is the morphological erosion and $\oplus$ the morphological dilation, is a granulometry. The random set $A$ can be characterized by recording, for distinct values of $\lambda$, the volume fraction of the residual set $A \backslash \Phi_{\lambda}(A)$. Granulometry measurements can be computed on the random $A$, as well as on its complementary set $A^{c}$.

In the remainder of the article, the quantities $m_{\mathscr{O}}$ and $m_{\Theta}$ will denote the statistical measurements as conducted on the observations and on the binary simulations, respectively. As discussed in section 1, the set of parameters $\Theta$ is fixed as to minimize the discrepancy between $m_{\mathscr{O}}$ and $m_{\Theta}$. This can be formulated as the following constrained optimization problem:

$$
\begin{aligned}
\hat{\Theta}:= & \arg \min _{\Theta}\left\|m_{\mathscr{O}}-m_{\Theta}\right\|_{2}^{2} \\
& \text { subject to } \Theta \in \mathscr{D}
\end{aligned}
$$

where $\mathscr{D}$ denotes the set of admissible parameters for the model. 


\section{BAYESIAN FORMULATION}

To determine a set of parameters, we propose in this article to rely on a Bayesian approach. Let us consider measurements obtained from a morphological model with parameters $\Theta$ and measurements conducted on the observation. By considering the quantity

$p(\mathscr{O} \mid \Theta)=\frac{1}{\sqrt{2 \pi|\Lambda|}} \exp \left(\left(m_{\mathscr{O}}-m_{\Theta}\right)^{T} \Lambda^{-1}\left(m_{\mathscr{O}}-m_{\Theta}\right)\right)$,

it is possible to define a notion of probability that the observations were obtained with the set of parameters $\Theta$. This quantity is indeed maximal when the measurements computed on realizations of the model exactly match the measurements computed on the observations. In Eq. (7), the covariance matrix $\Lambda$ is usually taken to be diagonal, and allows to assign a weight to the parameters corresponding to their respective importance. Others distributions can potentially be selected for the likelihood as long as the likelihood remains maximal when the simulated measurements match the observations. The choice of a Gaussian distribution is therefore mostly motivated by its simplicity.

It is also possible to define a prior distribution on the parameters $\Theta$. Usually, we are able, at least approximately, to define a range of variation for all parameters of the model:

$$
\Theta_{\min } \preceq \Theta \preceq \Theta_{\max },
$$

where $\preceq$ is a component-wise inequality. For instance, in the case of a Boolean model of disks with constant radii, the parameters of the model are the intensity $\theta$ of the underlying Poisson point process, as well as the radius $R$ of the disks:

$$
\Theta:=(\theta, R) .
$$

In this case, it is usually possible to estimate upper and lower bounds on these parameters. A simple way of defining a prior distribution is therefore to consider a uniform distribution between the lower and the upper bound for each parameter:

$$
p(\Theta):=\mathscr{U}_{\Theta_{\min }, \Theta_{\max }} .
$$

Using Bayes formula, it is possible to find an expression for the posterior distribution of the parameters $\Theta$ with respect to the observations $\mathscr{O}$ :

$$
p(\Theta \mid \mathscr{O}) p(\mathscr{O})=p(\mathscr{O} \mid \Theta) p(\Theta)
$$

Obviously, the prior probability law for the observation remains unknown. However, this quantity is independent from $\Theta$. Hence, we find that the posterior distribution is proportional to the likelihood multiplied by the prior distribution:

$$
p(\Theta \mid \mathscr{O}) \propto p(\mathscr{O} \mid \Theta) p(\Theta) .
$$

Starting from the posterior distribution of the parameters, several approaches can be considered to compute an optimal set of parameters. A first possibility is to maximize the posterior distribution with respect to the parameters $\Theta$. This can be for instance performed by using a projected gradient descent algorithm or any other classical optimization method.

\section{SAMPLING FROM THE POSTERIOR DISTRIBUTION}

In this section, we propose an alternative approach, which relies on a Monte Carlo Markov Chains (MCMC) algorithm to generate samples from the posterior distribution (Robert, 2004; Andrieu, 2003). This approach presents several advantages when compared to other methods including gradient descent. First, it is usually difficult to compute the gradient of the measurements with respect to the parameters, due to two main issues: there is usually no available analytical formula to compute the gradient, and the function $m_{\Theta}$ is a stochastic function of the set of parameters $\Theta$, which makes it difficult to estimate the gradient through a finite difference scheme. An additional advantage of the MCMC approach is that it allows to obtain samples that reproduce the actual posterior distribution, which enables us to capture some interesting features of the microstructure, including correlations between parameters or characteristic ranges of variation of the parameters.

In what follows, we rely on a Metropolis-Hastings algorithm to generate a sequence of samples from the posterior distribution. The algorithm works as follows:

- Initialization: A set of parameters is first generated from the prior distribution:

$$
\Theta_{1} \sim \mathscr{U}_{\Theta_{\min }} \Theta_{\max }
$$

- n-th iteration: At each iteration, we repeat the following steps:

1. Parameters sampling A set of parameters $\hat{\Theta}$ is generated from the current state $\Theta_{n}$ according to a proposal distribution $q$ :

$$
\hat{\Theta} \sim q\left(\hat{\Theta} \mid \Theta_{n}\right)
$$


2. Simulation A single simulation of the morphological model is computed with this set of parameters and a measurement vector $m_{\hat{\Theta}}$ is extracted from the microstructure computed with the model. The measurement vector is used to compute the posterior distribution, up to the constant factor $p(\mathscr{O})$ :

$$
p(\hat{\Theta} \mid \mathscr{O}) \propto p(\mathscr{O} \mid \hat{\Theta}) p(\hat{\Theta}) .
$$

3. Accept/reject step A quantity $r$ referred to as the Hastings ratio is computed:

$$
r=\min \left(1, \frac{p(\hat{\Theta} \mid \mathscr{O}) q\left(\Theta_{n} \mid \hat{\Theta}\right)}{p\left(\Theta_{n} \mid \mathscr{O}\right) q\left(\hat{\Theta} \mid \Theta_{n}\right)}\right)
$$

Noteworthy, the Hastings ratio $r$ is independent of the quantity $p(\mathscr{O})$. The new set of parameters $\hat{\Theta}$ is accepted or rejected with probability $r$. In practice, a random variable $u$ is drawn from the uniform distribution $\mathscr{U}(0,1)$, and the novel set of parameters is set according to the following formula:

$$
\Theta_{n+1}= \begin{cases}\hat{\Theta} & \text { if } u<r \\ \Theta_{n} & \text { otherwise }\end{cases}
$$

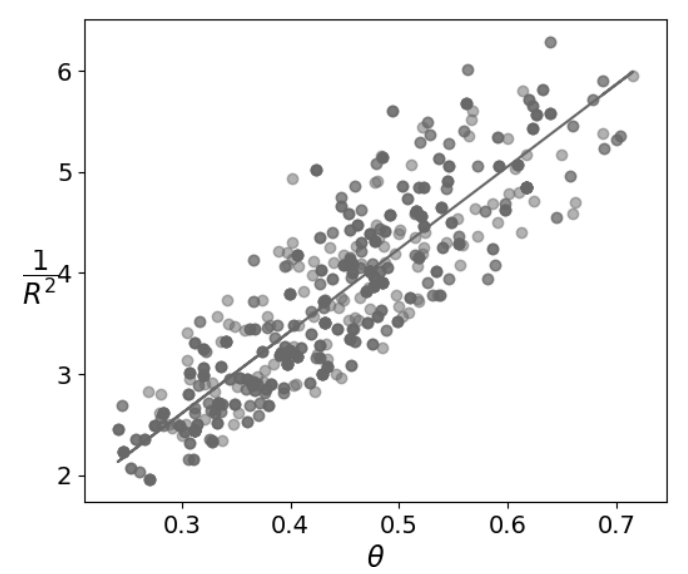

Fig. 1: Scatterplot of the parameters $1 / R^{2}$ and $\theta$ sampled by the Metropolis-Hastings algorithm for the Boolean model of disks. The color intensity of each points corresponds to the frequency of the corresponding parameters within the samples

\section{NUMERICAL EXPERIMENTS}

\section{BOOLEAN MODEL OF DISKS WITH CONSTANT RADII}

We first illustrate our optimization approach on a purely numerical example, where a set of $N=10$ random microstructures are generated in a domain of size 30 by 30 in $\mathbb{R}^{2}$ using a Boolean model of disks. The intensity of the Boolean model is taken equal to 0.45 , and the radius of the disks to 0.5 in this specific example. A sample of the simulated microstructure is displayed in Fig. 2. The covariance, the granulometry and the granulometry of the complementary are computed and averaged for each one of the microstructures to yield a measurement vector $m_{\mathscr{O}}$.

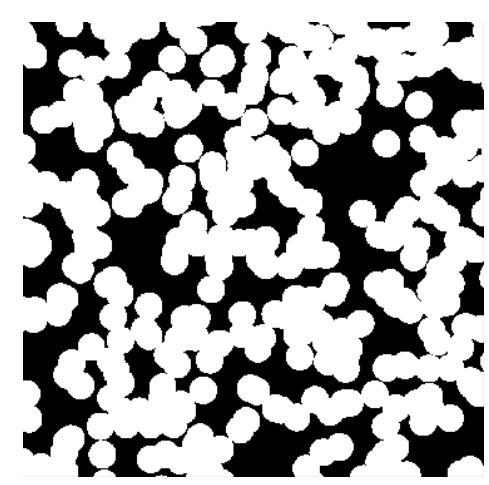

Fig. 2: Sample of the microstructure generated with the Boolean model of disks

The upper and lower bounds for the model parameters used to define the prior distribution are indicated in table 1 . The covariance matrix $\Lambda$ used to define the covariance in the likelihood function (7) is taken to be diagonal. However, to put more emphasis on the correlations at small scale, we rely on the following expression for the coefficients of $\Lambda$ corresponding to the covariance measurements:

$$
\Lambda_{n, n}=\lambda\left(1+\frac{1}{n}\right)^{-1},
$$

where $\Lambda_{n, n}$ is the diagonal coefficient of $\Lambda$ corresponding to the $n$-th covariance measurement $C(n \Delta h)$ and $\lambda>0$. The variance of the covariance measurements is set uniformly equal to $\lambda$.

We initialize the Metropolis-Hastings algorithm by sampling a first set of parameters $\Theta_{1}:=\left(\theta_{1}, R_{1}\right)$ directly from the prior distribution. We select $\lambda=0.1$ in our computations. At each step of the algorithm, the proposal distribution for each parameter is defined by the truncated normal distribution:

$$
q\left(\theta \mid \theta_{n}\right)=\mathscr{N}_{t r}\left(\theta_{n}, \sigma_{\theta}, \theta_{\min }, \theta_{\max }\right),
$$

where $\theta_{\min }$ (resp. $\theta_{\max }$ ) is the minimal (resp. maximal) possible value of the parameter, and the standard deviation $\sigma$ is set to:

$$
\sigma_{\theta}=\frac{\theta_{\max }-\theta_{\min }}{\Delta}
$$

The parameter $\Delta$ in this equation must be carefully selected. By decreasing the value of $\Delta$, we enable 


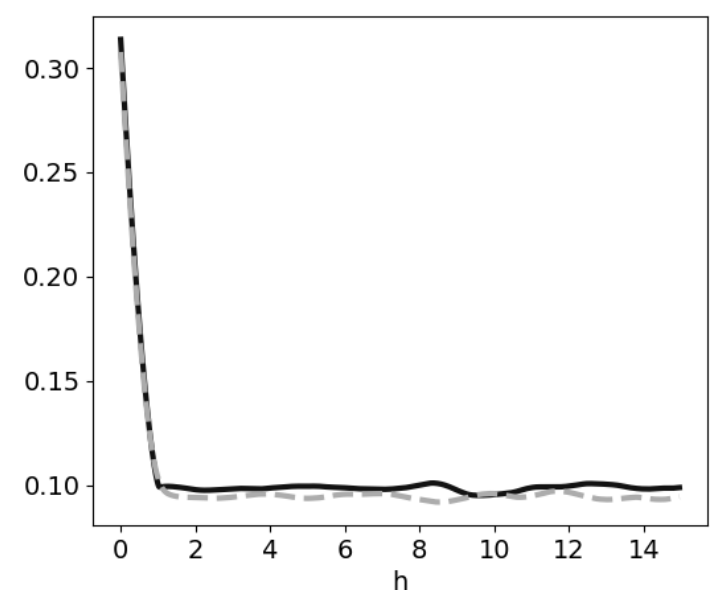

(a) Covariance
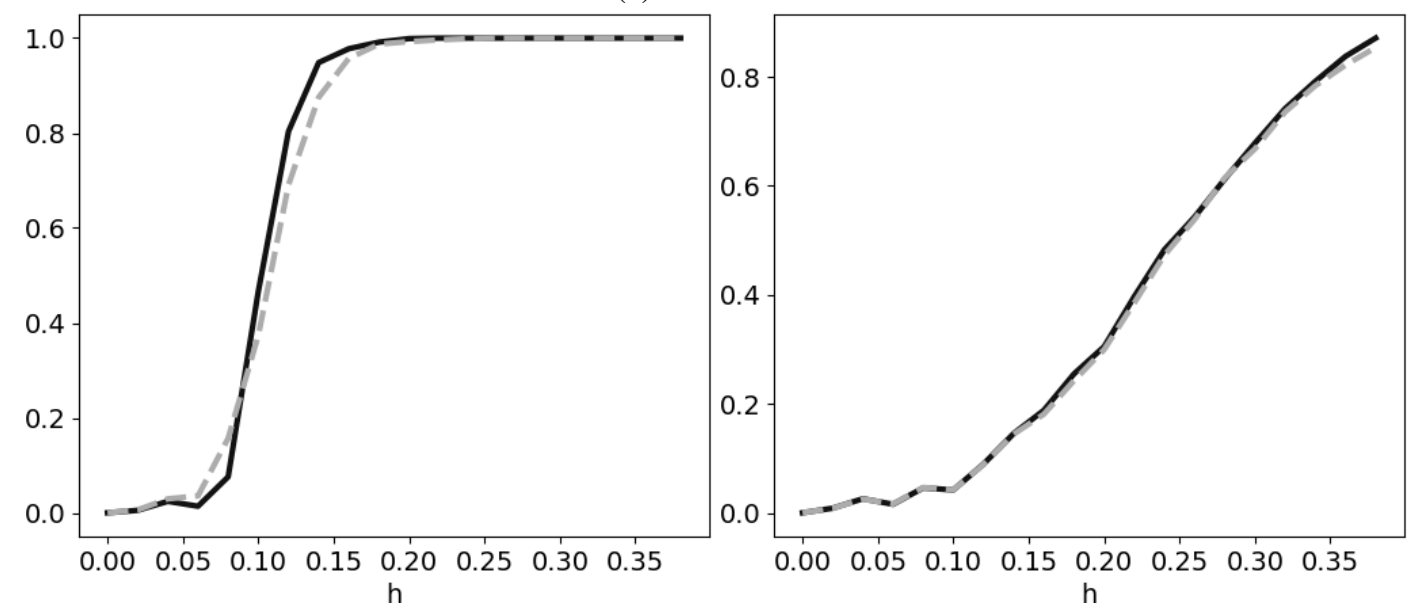

(b) Granulometry measurements of the microstructure (left) and of the complementary (right)

Fig. 3: Comparison between the experimental measurements and the measurements obtained with simulations conducted with the MAP parameters identified by the MCMC algorithm for the Boolean model of disks .

proposals that are far from the current state, therefore reducing the acceptation rate. By contrast, increasing the value of $\Delta$ leads to proposals that are closer to the current state, at the detriment of the exploration properties of the algorithm. Here, we fix $\Delta=20$ to get an acceptation rate of 0.3 in the algorithm.

We stop the algorithm after 500 iterations, and we discard the first 100 parameters sampled by the algorithm. We estimate the set of optimal parameters for the microstructure by considering the maximal a posteriori value found for the sampled parameters:

$$
\hat{\Theta}:=\arg \max _{n \in\{100, \ldots, 500\}} p\left(\Theta_{n} \mid \mathscr{O}\right) .
$$

In our experiment, we found the estimation of the models parameters to be already accurate after around 200 sampling iterations, and drawing additional samples only improve marginally the estimation. The optimal set of parameters $\Theta$ found by the MetropolisHastings algorithm for the Boolean model of disks are displayed in Tab. 1, along with the standard deviation of the sampled parameters. The standard deviation yields a useful information regarding the sensibility of the parameters with respect to the Bayesian model. We can note that there is a good agreement between the parameters identified by the algorithm and the ones corresponding to the experimental microstructure. A comparison between the measurements as conducted on the experimental microstructure and as obtained with the optimal set of parameters $\hat{\Theta}$ is displayed in Fig. 3.

\begin{tabular}{l|ll} 
& $\theta\left[L^{-1}\right]$ & $R[L]$ \\
\hline Target & 0.45 & 0.5 \\
$\hat{\Theta}$ & $0.44 \pm 0.05$ & $0.52 \pm 0.025$ \\
Min. Val. & 0.2 & 0.15 \\
Max. val. & 0.9 & 1.05
\end{tabular}

Table 1: Optimal parameters found by the MetropolisHastings algorithm for the Boolean model of disks 


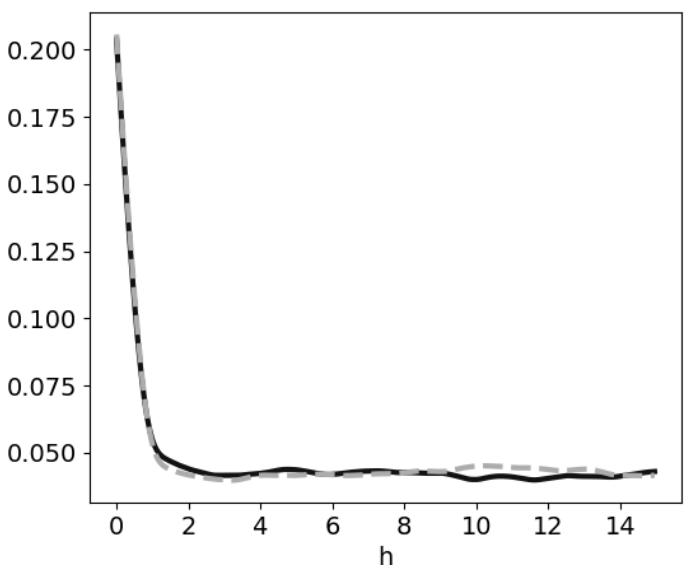

(a) Covariance
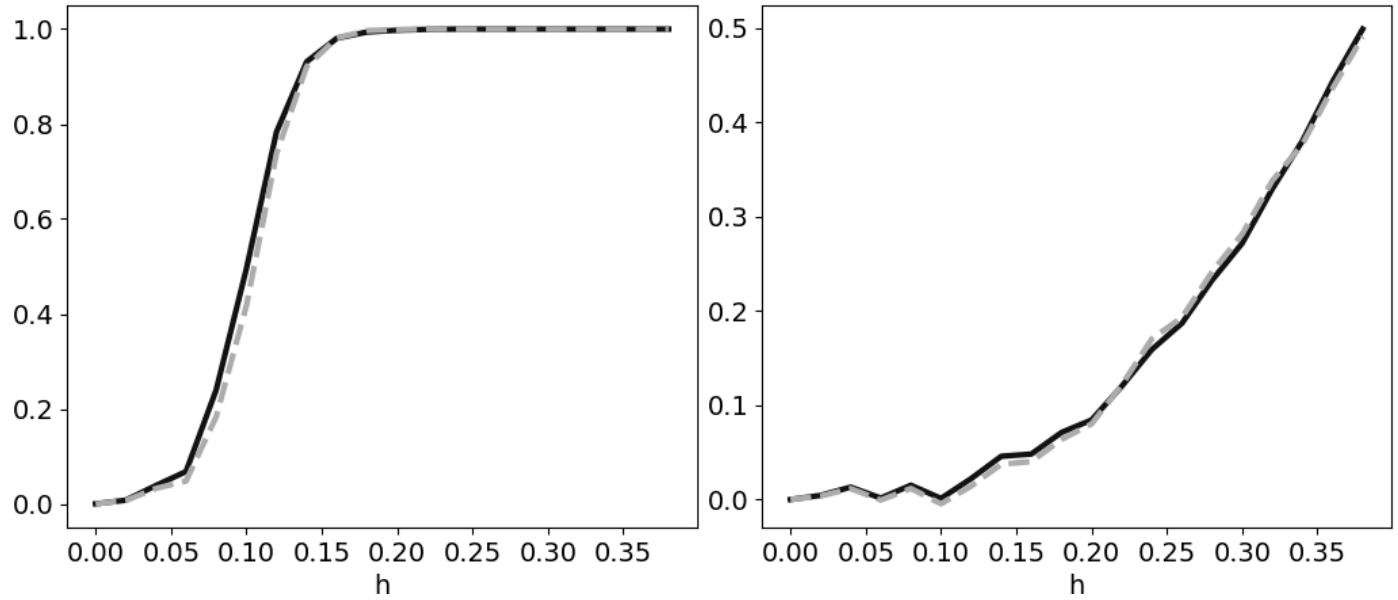

(b) Granulometry measurements of the microstructure (left) and of the complementary (right)

Fig. 4: Comparison between the experimental measurements and the measurements obtained with simulations conducted with the MAP parameters identified by the MCMC algorithm for the Cox-Boolean model of disks .

Fig. 1 displays a scatterplot of the parameters sampled by the Metropolis-Hastings algorithm. Interestingly, we note that the parameters $\theta$ and $R$ sampled by the algorithm are strongly correlated. We performed a linear regression between the parameters $\theta$ and $1 / R^{2}$, to find a coefficient of determination equal to 0.81 . For a Boolean model of disks, the volume fraction $v$ is known to be given by

$$
v=1-\exp \left(\pi R^{2} \theta\right) .
$$

This leads to the linear relationship

$$
\frac{1}{R^{2}}=-\frac{\pi}{\log (1-v)} \theta .
$$

The covariance measurements are highly sensitive to the volume fraction. Hence, by construction, the Metropolis-Hastings will usually select a set of parameters that allows to obtain a volume fraction similar to the one measured on the experimental samples. As evidenced in Fig. 1, the parameters are indeed distributed around a straight line.

\section{COX-BOOLEAN MODEL OF DISKS}

In this section, we illustrate the optimization approach on a more complicated numerical example, where a set of $N=10$ random microstructures are generated in $\mathbb{R}^{2}$ using a Cox-Boolean model of disks. In this example, the microstructure is composed of two scales. A first scale defines exclusion zones that remains empty of any inclusions. These exclusion zones are modelled by a Boolean model of disk with constant radii. The intensity $\theta_{e}$ of the Boolean model is taken equal to 0.1 , and the radius $R_{e}$ of the disks to 1 in this specific example. The second scale simulates the inclusions of the microstructure, and is modelled by a Boolean model of disks with intensity $\theta=0.45$. The radii of the disks are sampled according to a normal distribution with mean $R$ equal to 0.5 and standard deviation 0.1. A sample of the simulated microstructure is displayed in Fig. 5. As in the previous example, the covariance, the granulometry and the granulometry of the complementary set are computed 
and averaged for each one of the microstructures to yield a measurement vector $m_{\mathscr{O}}$.

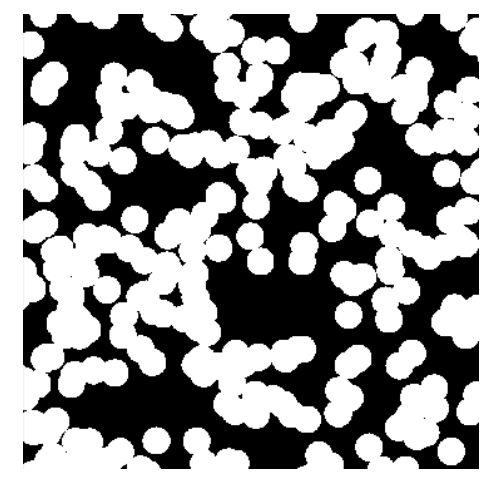

Fig. 5: Sample of the microstructure generated with the Cox-Boolean model of disks

The upper and lower bounds for the model parameters used to define the prior distribution are indicated in table 2 . The covariance matrix $\Lambda$ used to define the covariance in the likelihood function 7 is the same as the one used in the previous example, as well as the proposal distribution. Again, we initialize the Metropolis-Hastings algorithm by sampling a first set of parameters directly from the prior distribution and we fix the value of $\lambda$ to 0.1 in the simulation. We stop the algorithm after 500 iterations, and we discard the first 100 parameters sampled by the algorithm. We estimate the set of optimal parameters for the microstructure by considering the sample exhibiting the maximal a posteriori probability (MAP) within all sampled parameters.

The optimal set of parameters $\hat{\Theta}$ found by the Metropolis-Hastings algorithm for the Cox-Boolean model of disks is displayed in Tab. 2. Again, we can note that there is a good agreement between the parameters identified by the algorithm and the ones corresponding to the experimental microstructure. Noteworthy, due to the smaller influence of the parameters $R_{e}$ and $\theta_{e}$, the estimation error is larger for these parameters. A comparison between the measurements as conducted on the experimental microstructure and as obtained with the optimal set of parameters $\hat{\Theta}$ is displayed in Fig. 4.

\begin{tabular}{l|llll} 
& $\theta_{e}\left[L^{-1}\right]$ & $R_{e}[L]$ & $\theta\left[L^{-1}\right]$ & $R$ \\
\hline Target & 0.1 & 1. & 0.45 & 0.5 \\
$\hat{\Theta}$ & 0.13 & 0.85 & 0.45 & 0.5 \\
Std. Dev. & 0.03 & 0.25 & 0.05 & 0.04 \\
Min. Val. & 0.05 & 0.5 & 0.2 & 0.15 \\
Max. val. & 0.2 & 2. & 0.9 & 1.05
\end{tabular}

Table 2: Optimal parameters found by the MetropolisHastings algorithm for the Cox-Boolean model of disks

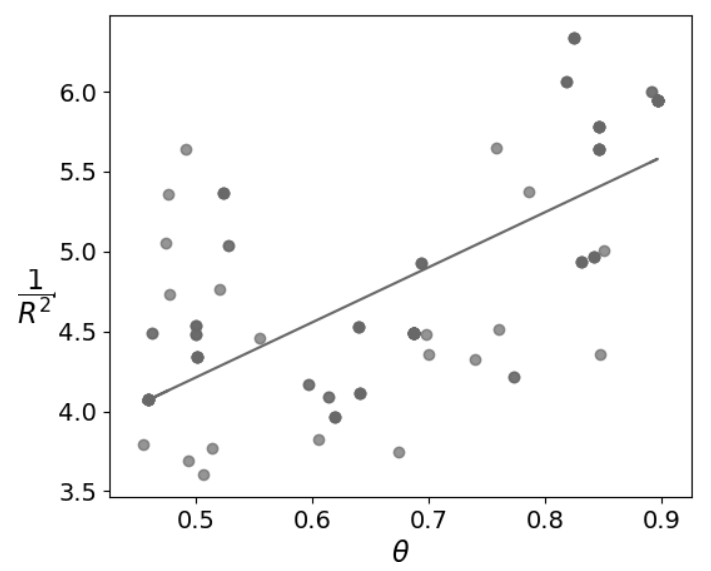

Fig. 6: Scatterplot of the parameters $1 / R^{2}$ and $\theta$ sampled by the Metropolis-Hastings algorithm for the Cox-Boolean model of disks

It is also interesting to use the sampled parameters to determine if there is still a linear correlation between the values sampled for $1 / R^{2}$ and $\theta$. Fig. 6 displays a scatterplot of the parameters sampled by the Metropolis-Hastings algorithm. For the Cox-Boolean model, we note that the parameters $\theta$ and $R$ sampled by the algorithm remain correlated, but the correlation is less obsvious than for the Boolean model. A linear regression performed between the parameters $\theta$ and $1 / R^{2}$ yields a coefficient of determination equal to 0.51 . Due to the presence of exclusion zones in the microstructure, Eq. (23) is not valid anymore, which explains this smaller value.

\section{EXPERIMENTS}

In this last section, we present an application of the proposed optimization approach to an experimental microstructure. The corresponding material is a colloid film constituted of pigments embedded in a matrix. The pigments have an elongated ellipsoidal form close to the one of a needle. A dataset of experimental images of the microstructure was obtained using transmission electron microscopy (TEM) imaging. An example is shown in Fig 8. We can note on the experimental TEM images that the pigments tend to aggregate in the microstructure, therefore forming clusters whose characteristic size significantly surpasses the size of the pigments.

To describe the 3D microstructure, we rely on a two-scale model. The model is as follows:

- The first scale of the simulation aims at describing the pigments aggregates. We describe these aggregates by considering a Boolean model of spheres with constant radius $R_{i}$. The model describing the aggregates is therefore characterized by two parameters, namely the 


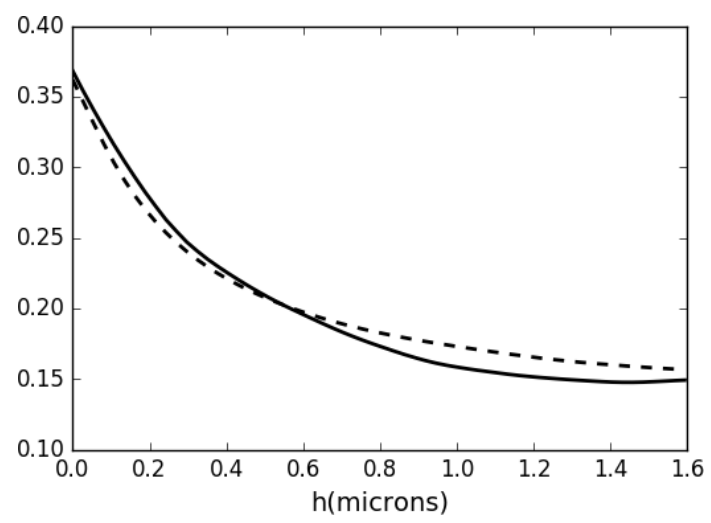

(a) Covariance

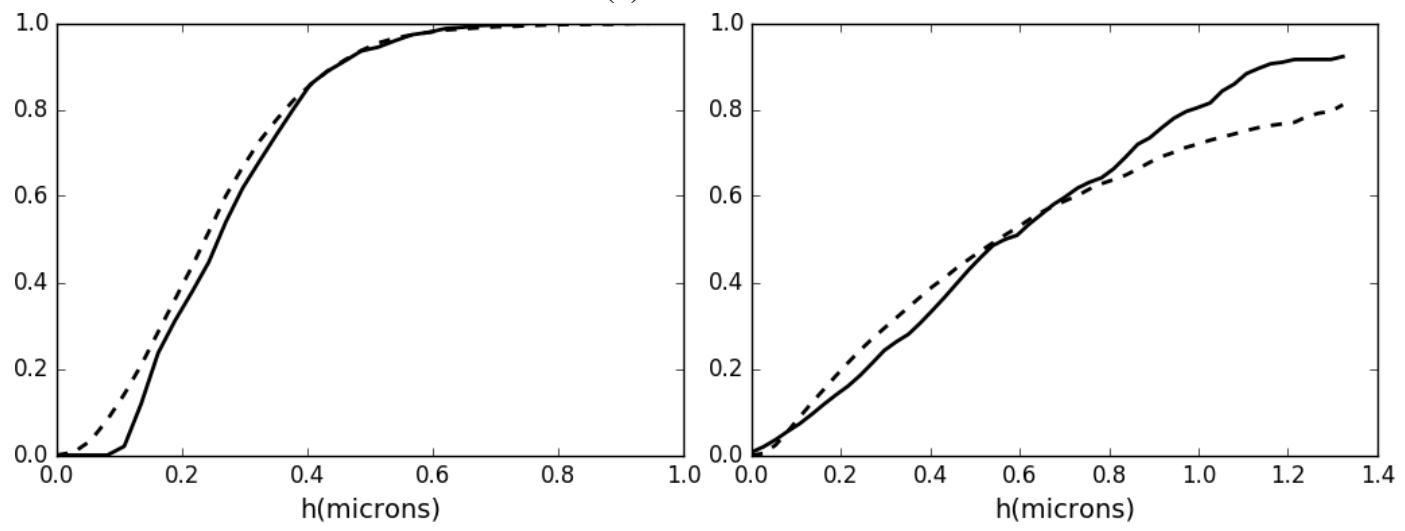

(b) Granulometry measurements

Fig. 7: Comparison between the covariance and the granulometries as obtained on the experimental TEM images (dashed lines) and on the simulated TEM images for the "needles" microstructure.
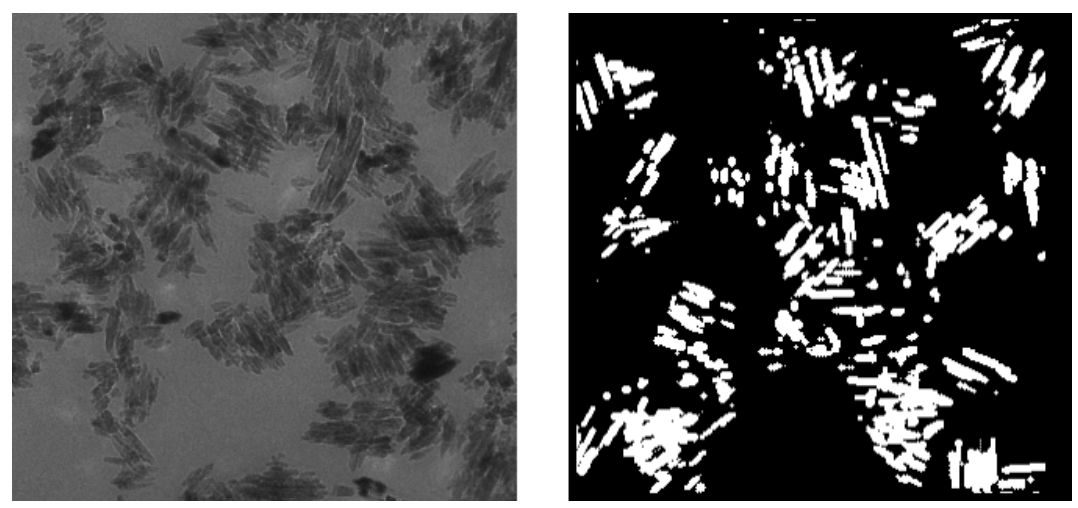

Fig. 8: Needles microstructure: experimental TEM image (left) and binary image simulated with the morphological model using the optimal set of parameters identified by the Bayesian approach

\begin{tabular}{l|c|c|c|c|c|}
$\theta_{i}\left(\mu \mathrm{m}^{-3}\right)$ & $R_{i}(\mu \mathrm{m})$ & $\theta\left(\mu \mathrm{m}^{-3}\right)$ & $L(\mu \mathrm{m})$ & $l(\mu \mathrm{m})$ & $h(\mu \mathrm{m})$ \\
\hline 0.56 & 0.54 & 45. & $0.56 \pm 0.08$ & $0.07 \pm 0.01$ & 0.2
\end{tabular}

Table 3: Optimal parameters for the "needles" microstructure 
intensity $\theta_{i}$ of the Poisson point $\mathscr{P}$ process used to construct the Boolean model and the radii $R_{i}$ of the spheres. The first scale therefore correspond to the geometrical set defined by the union

$$
\mathscr{S}=\cup_{i \in I} \mathscr{S}_{i},
$$

where $\mathscr{S}_{i}$ is the sphere implanted on the i-th point of the process.

- The second scale of the model simulates the pigments within the aggregates. The pigments are represented by a Boolean model of ellipsoids whose largest semi-axis follows a normal law with mean $L$ and standard deviation $\sigma_{L}$, and whose smallest semi-axes follow a normal law with mean $l$ and standard deviation $\sigma_{l}$. An hardcore distance $h$ is introduced in the underlying point process. For each sphere $\mathscr{S}_{i}$, the ellipsoids are implanted at locations sampled from a Poisson point process with intensity $\theta$ restricted to the sphere. In each sphere $\mathscr{S}_{i}$, a single orientation for the ellipsoids is sampled uniformly from Euler's angles.

Overall, the model for describing the microstructure requires eight parameters, namely the intensity $\theta_{i}$ of the Poisson point process for the aggregates, their radius $R_{i}$, the semi-axes lengths $L$ and $l$ of the pigments, along with the corresponding standard variations $\sigma_{L}$ and $\sigma_{l}$, the intensity $\theta$ of the point process used for the pigments and the hardcore distance $h$.

To determine the model parameters, we rely on the Bayesian approach described in the previous section. We initialize the Metropolis-Hastings algorithm with a value of $\lambda$ equal to 1 in the simulation, and we stop the algorithm after 200 iterations. As previously, we estimate the set of optimal parameters for the microstructure by considering the sample exhibiting the maximal a posteriori probability (MAP) within all sampled parameters. During the MCMC run, we simulate TEM images of the microstructure by first generating a 3D sample of the model, then extracting a thick slice of the 3D volume whose thickness matches the one of the experimental slices used to obtain the TEM images and finally projecting the thick slice on a single plane. An illustrative example of the microstructure generated with this approach is presented in Fig. 8. Covariance and granulometry measurements are finally conducted on the experimental and simulated TEM images.

The optimal set of parameters $\hat{\Theta}$ found by the Metropolis-Hastings algorithm for the morphological model is displayed in Tab. 3. A comparison between the measurements as conducted on the experimental microstructure and as obtained with the optimal set of parameters is displayed in Fig. 7. Again, we can note that the optimal set of parameters allows us to obtain a good match between the experimental measurements and the measurements conducted on simulations of the morphological models.

\section{CONCLUSION}

In this article, we proposed a Bayesian approach to determine the parameters of morphological models of microstructure based upon measurements conducted on experimental images of the microstructure. We demonstrate on several examples that this approach allows us to properly identify the optimal parameters of distinct morphological models and to identify potential correlations between the parameters of the models. The proposed methodology present several advantages when compared to other approaches used to perform the parameters determination. In particular, it does not require the computation of the gradient of the model with respect to the parameters, and it yields a sequence of samples of parameters, which allows to quantify the sensibility of the parameters to the model and to highlight potential correlation between parameters of the model.

\section{REFERENCES}

Andrieu C, De Freitas N, Doucet A and Jordan M (2003). An introduction to MCMC for machine learning. Mach learn, 50(1):5-43.

Belhadj J, Romary T, Gesret A, Noble M and Figliuzzi B (2018). New parameterizations for Bayesian seismic tomography. Inverse Probl 34(6):065007

Bortolussi V, Figliuzzi B, Willot F, Faessel M, Jeandin M (2018). Morphological modeling of cold spray coatings. Image Anal Stereol 37(2):145-58

Chiu SN, Stoyan D, Kendall WS and Mecke J (2013). Stochastic geometry and its applications. John Wiley \& Sons

Figliuzzi B, Jeulin D, Faessel M, Willot F, Koishi $M$ and Kowatari N (2016). Modelling the microstructure and the viscoelastic behaviour of carbon black filled rubber materials from 3D simulations. Tech Mechanik 32(1-2):22-46

Figliuzzi B (2019). Eikonal-based models of random tessellations. Image Anal Stereol 38(1):15-23.

Gasnier J-B, Willot F, Trumel H, Figliuzzi B, Jeulin D and Biessy M (2015). A Fourier-based numerical homogenization tool for an explosive material. Matériaux \& Techniques 103(3):308 
Jeulin D (1991). Modèles morphologiques de structures aléatoires et de changement d'échelle. Doctoral dissertation, Caen

Jeulin D (2012). Morphology and effective properties of multi-scale random sets: A review. Comptes Rendus Mécanique 340(4-5):219-29

Jeulin D (2017). Morphological models. In: Encyclopedia of Continuum Mechanics. Springer, $1-12$

Jeulin D (2021). Morphological Models of Random Structures. Springer International Publishing.

Koishi M, Kowatari N, Figliuzzi B, Faessel M, Willot $F$ and Jeulin D (2017). Computational material design of filled rubbers using multi-objective design exploration. 10th ECCMR

Moreaud M, Jeulin D, Morard V and Revel R (2012). TEM image analysis and modelling: application to boehmite nanoparticles. J MicroscOxford 245(2):186-99

Moussaoui H, Laurencin J, Gavet Y, Delette G, Hubert M, Cloeten P and Debayle J (2018).
Stochastic geometrical modeling of solid oxide cells electrodes validated on 3D reconstructions. Comp Mater Sci 143:262-76

Moussaoui H, Sharma R K, Debayle J, Gavet Y, Delette G and Laurencin J (2019). Microstructural correlations for specific surface area and triple phase boundary length for composite electrodes of solid oxide cells. J Power Sources 412:736-48.

Ohser J and Schladitz K (2009). 3D images of materials structures: processing and analysis. John Wiley \& Sons

Robert C and Casella G (2004). Monte Carlo statistical methods. New York: Springer.

Torquato S (2013). Random heterogeneous materials: microstructure and macroscopic properties. Springer Science \& Business Media

Wang H, Pietrasanta A, Jeulin D, Willot F, Faessel M, Sorbier L and Moreaud M (2015). Modelling mesoporous alumina microstructure with 3D random models of platelets. J Microsc-Oxford 260(3):287-301 